EPJ Web of Conferences 73, 06004 (2014)

DOI: $10.1051 /$ epjconf/20147306004

(C) Owned by the authors, published by EDP Sciences, 2014

\title{
Electron-deuteron scattering based on the Chiral Effective Field Theory
}

\author{
Dagmara Rozpȩdzik ${ }^{\mathrm{a}}$
}

M. Smoluchowski Institute of Physics, Jagiellonian University, 30059 Kraków, Poland

\begin{abstract}
Based on the Chiral Effective Field Theory (ChEFT) dynamical picture of the two-pion exchange (TPE) contributions to the nuclear current operator which appear at higher order chiral expansions were considered. Their role in the electrondeuteron scattering reactions was studied and chiral predictions were compared with those obtained in the conventional framework. Results for cross section and various polarization observables are presented. The bound and scattering states were calculated with five different chiral nucleon-nucleon (NN) potentials which leads to the so-called theoretical uncertainty bands for the predicted results.
\end{abstract}

\section{Introduction}

Chiral effective field theory (ChEFT) is a modern framework to analyze properties of few-nucleon systems, especially at low energies. This approach is a powerful tool for the derivation of the nuclear forces. For energies below the pion-production threshold it is possible to eliminate the pionic degrees of freedom and derive nuclear potentials and nuclear current operators solely in terms of nucleonic degrees of freedom $[1,2]$. This is very important because the consistence between two-nucleon forces, many-nucleon forces and corresponding current operators has been not yet achieved, despite a lot of experience gained in the past.

Electron scattering is used for obtaining information on many important properties of nuclei. At present electron scattering is studied with the aim to probe the nuclear wave functions and obtain information about the nuclear current operator. In this context, the few-body systems $(A \leq 3)$ are particularly important since the two- and three-nucleon bound and scattering states, at least nonrelativistically, can be calculated accurately for any given two- and three-body interactions.

Experimental studies of inelastic electron-deuteron scattering inspired many theoretical efforts and have greatly contributed to our understanding of the nucleon-nucleon interaction and the role of the meson exchange current or relativistic corrections in the nuclear systems. Recent review article [3] illustrate the present state of knowledge on the structure of the deuteron and, more generally, on the dynamical picture of the electron-deuteron scattering reactions.

In the two-nucleon $(2 \mathrm{~N})$ system, the leading contributions to the exchange current originate from one-pion exchange and are well known [1]. The corrections to the $2 \mathrm{~N}$ current operator from the two-pion

\footnotetext{
ae-mail: dagmara.rozpedzik@uj.edu.pl
}

This is an Open Access article distributed under the terms of the Creative Commons Attribution License 4.0, which permits unrestricted use, distribution, and reproduction in any medium, provided the original work is properly cited. 
exchange $2 \mathrm{~N}$ current operator were derived using the method of unitary transformation [2]. The resulting current operator is consistent with the corresponding chiral two-nucleon potential obtained within the same scheme. In the present work, the effects of the leading two-pion exchange $2 \mathrm{~N}$ operator in the deuteron electrodisintegration reaction are explored. It has to be emphasized, however, that the presented calculation is not yet complete. In particular, the corresponding expressions for the one-pion exchange (OPE) and short-range contributions to the current operator within the method of unitary transformation are not yet included. The important outcome of the presented work is that various observables in the deuteron electrodisintegration appear to be highly sensitive to the two-pion exchange current.

\section{Formalism}

The formalism to describe $2 \mathrm{~N}$ electromagnetic reactions requires the knowledge of the consistent potential and electromagnetic current. The NN potential based on ChEFT is well known up to nextto-next-to-leading (N2LO) order in the chiral expansion. As already pointed out, this study is restricted to next-to-leading order (NLO) long-range contributions to the $2 \mathrm{~N}$ current operator. To achieve a better accuracy, all calculations are made using the N2LO potential.

The effective current operator for the $2 \mathrm{~N}$ system is a sum of the single-nucleon operators $J_{\text {single }}^{\mu}(i), i=1,2$ and two-nucleon operators of different type $\left(J^{\mu}(1,2)\right)[4]$ :

$$
J_{2 N}^{\mu}=J_{\text {single }}^{\mu}(1)+J_{\text {single }}^{\mu}(2)+J_{\text {ope }}^{\mu}(1,2)+J_{\text {tpe }}^{\mu}(1,2)+J_{\text {cont }}^{\mu}(1,2),
$$

where the expressions for the single-nucleon and OPE currents are well established. The $J_{t p e}^{\mu}(1,2)$ and $J_{\text {cont }}^{\mu}(1,2)$ denote TPE contributions and contact terms of nuclear current, respectively.

In this paper, we concentrate on a treatment of the long-range TPE contributions to the $2 \mathrm{~N}$ current operator derived in Ref. [2]. The expressions for the TPE current operator in momentum space are complex and contain the standard loop functions and the three-point functions in a form suitable for numerical calculations.

The TPE four-current operator $J_{t p e}^{\mu}=\left(J_{t p e}^{0}, \vec{J}_{\text {ope }}\right)$ can be decomposed according to its isospin-spinmomentum structure and quite generally written in the form:

$$
J_{\text {tpe }}^{0}=\sum_{\alpha=1}^{5} \sum_{\beta=1}^{8} f_{\alpha}^{\beta S}\left(\vec{q}_{1}, \vec{q}_{2}\right) T_{\alpha} O_{\beta}^{S}, \quad \vec{J}_{\text {tpe }}=\sum_{\alpha=1}^{5} \sum_{\beta=1}^{24} f_{\alpha}^{\beta}\left(\vec{q}_{1}, \vec{q}_{2}\right) T_{\alpha} \vec{O}_{\beta},
$$

where $\vec{q}_{i} \equiv \vec{p}^{\prime}-\vec{p}$ is the momentum transferred to nucleon $i, T_{\alpha}$ is the $2 \mathrm{~N}$ isospin operator, $O_{\beta}^{S}$ and $\vec{O}_{\beta}$ are the (momentum dependent) spin operators in the $2 \mathrm{~N}$ space, $f_{\alpha}^{\beta S}$ and $f_{\alpha}^{\beta}$ are scalar functions. The explicit form of the scalar functions can be found in Ref. [2].

One-photon exchange approximation was used, in which the scattering amplitude is written as a contraction of the electron part with the nuclear matrix element supplemented by the virtual photon propagator:

$$
M=-\frac{e^{2}}{\mathbf{q}^{2}}\left[\bar{u}\left(k^{\prime}, h^{\prime}\right) \gamma_{\mu} u(k, h)\right]\left\langle\Psi_{\text {scatt }}\left|J_{2 N}^{\mu}(\vec{Q})\right| \Psi_{\text {bound }}\right\rangle,
$$

where matrix elements are so-called nuclear matrix elements $N^{\mu}$. The expressions for both the photon propagator and the so-called "electron arm" are analytical known. In the electron part, one finds the Dirac spinors representing the electron in the initial state (with the four-momentum $k$ and the helicity $h$ ) and in the final state (with the four-momentum $k^{\prime}$ and the helicity $h^{\prime}$ ) as well as the standard $\gamma^{\mu}$-matrix [5].

Deuteron electrodisintegration has been studied systematically for many years using the traditional approach [6]. The special emphasis has been put on polarization observables focusing on the role of the meson exchange currents, short-range interactions and various relativistic effects. In this work the 
Table 1. Selected kinematical configurations, where $E, E^{\prime}, \theta_{e}, Q$ and $\omega$ denote the initial and final electron energies, electron scattering angle, three-momentum transfer and energy transfer, respectively.

\begin{tabular}{lccccc}
\hline Kinematics & $E[\mathrm{MeV}]$ & $\theta_{e}[\mathrm{deg}]$ & $E^{\prime}[\mathrm{MeV}]$ & $\mathrm{Q}[\mathrm{MeV} / \mathrm{c}]$ & $\omega[\mathrm{MeV}]$ \\
\hline KIN1 & 62 & 70 & 35 & 60 & 27 \\
KIN2 & 105 & 70 & 52 & 100 & 50 \\
KIN3 & 140 & 30 & 50 & 100 & 90 \\
\hline
\end{tabular}
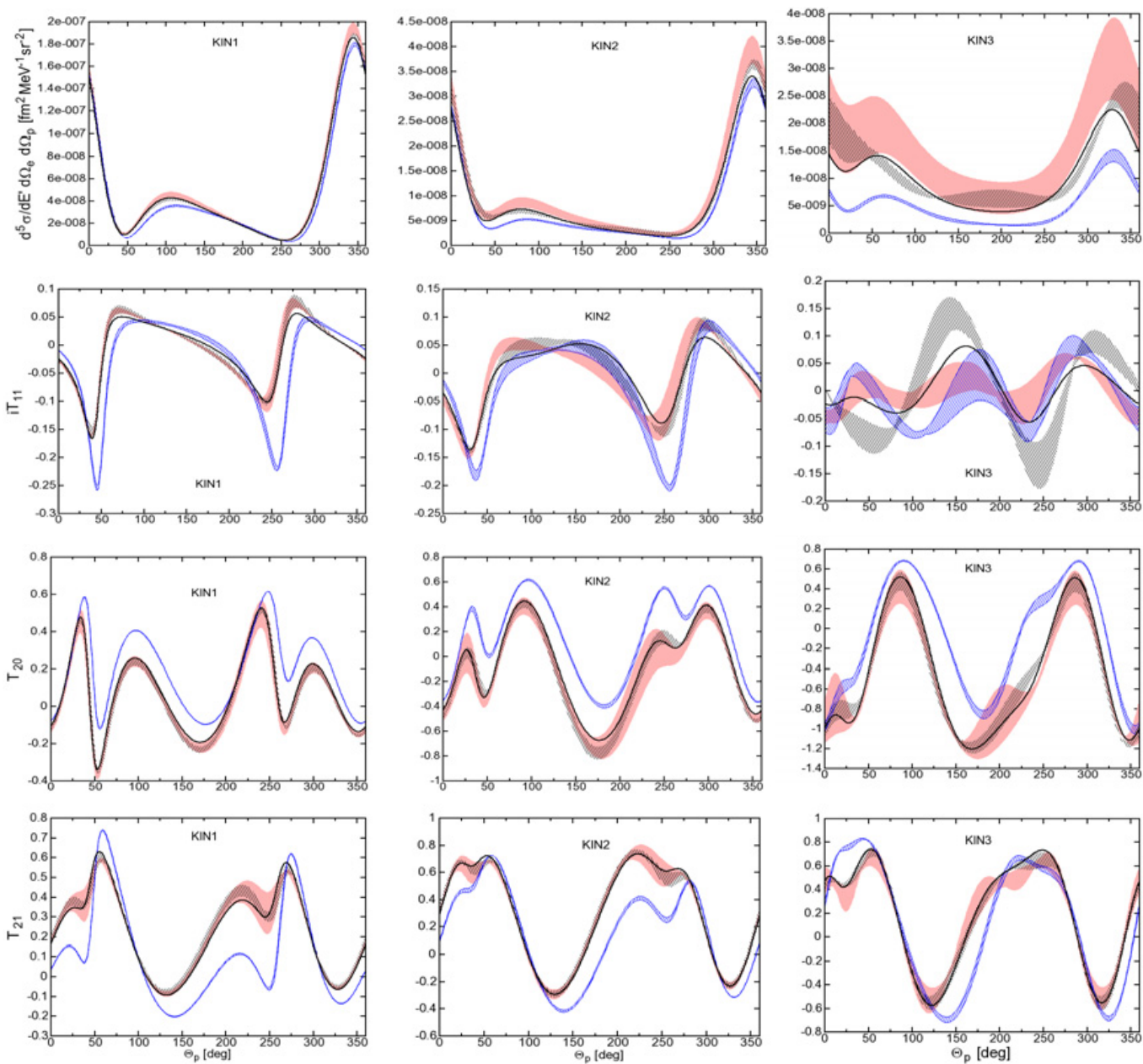

Figure 1. Differential cross section, deuteron tensor analyzing powers $i T_{11}$ (second row), $T_{2 i}, i=0,1$ (lower rows) as a function of outgoing proton angle $\theta_{p}$ for KIN1-KIN3 (Table 1). The blue band covers results obtained with the single-nucleon current only, the dark band represents predictions based on the single-nucleon and OPE parts, and the pink band contains additionally TPE parts. The black line represents predictions obtained with AV18 potential.

dynamical ingredients derived within ChEFT are employed as consistently as possible and the focus is on the nuclear current operator. Nuclear matrix elements for inelastic electron-deuteron scattering are specified as the charge density matrix element:

$$
N^{0}=\left\langle\Psi_{\text {scatt }}\left|J_{\text {single }}^{0}(\vec{Q})+J_{\text {tpe }}^{0}(\vec{Q})\right| \Psi_{\text {deut }}\right\rangle,
$$


and the matrix elements of the vector parts of the current operator:

$$
\vec{N}=\left\langle\Psi_{\text {scatt }}\left|\vec{J}_{\text {single }}(\vec{Q})+\vec{J}_{\text {ope }}(\vec{Q})+\vec{J}_{\text {tpe }}(\vec{Q})\right| \Psi_{\text {deut }}\right\rangle,
$$

where the individual parts, $\vec{J}_{\text {single }}(\vec{Q}), \vec{J}_{\text {ope }}(\vec{Q})$ and $\vec{J}_{\text {tpe }}(\vec{Q})$ are defined in Eq. (2). The formalism for calculations of the nuclear matrix elements in momentum space employs the standard partial wave decomposition of the chiral potential as described in Ref. [4].

\section{Results}

Resulting observables obtained for selected kinematics listed in Table 1 are presented in Fig. 1. For small values of $Q$ and $\omega$, for most observables a good agreement between MEC results and AV18 predictions has been found. With increasing $Q$, however, the width of the prediction bands increases. It can be noticed that the predictions strongly depend on $\omega / Q$ ratio, and significantly worsen when $\omega$ is close to $Q(\omega / Q \approx 1)$. This can be especially seen in the cross section, $T_{21}$ and $T_{22}$. For low $Q$ values, the bands are narrower, the predictions are more accurate and agree with AV18. As for now, there are insufficient experimental data for low $Q$ values, thus it is impossible to decide which picture, ChEFT or AV18 is better and whether adding TPE current is significant for proper description of the observables. In case of inclusive cross section, for small $Q$ value, no contributions from MEC currents has been seen. For higher $Q$, MEC contributions are very small and noticeable only for small values of the nucleon-proton energy.

\section{Conclusions}

The effects of the TPE currents derived recently in the framework of ChEFT in the deuteron electrodisintegration reaction were presented. The influence of various ingredients of the chiral $2 \mathrm{~N}$ current operator on the unpolarized cross section and several (un)polarization observables has been shown. As a main outcome of the study, it was shown that the new terms in the exchange current operator beyond the well-known one-pion exchange contribution play an important role in electrodisintegration. The dynamical framework is still in development. The inclusion of the consistent short-range contact and higher-order OPE contributions to the $2 \mathrm{~N}$ current operator is in progress.

The author would like to thank E. Epelbaum and S. Kölling for their input into the presented work. The project has been supported by the grant of the Polish Ministry of Science and Higher Education No. 7150/E-338/M/2013.

\section{References}

[1] E. Epelbaum, Prog. Part. Nucl. Phys. 57, 654 (2006)

[2] S. Kölling, E. Epelbaum, H. Krebs, Ulf-G. Meißner, Phys. Rev. C80, 045502 (2009)

[3] H. Arenhövel, W. Leidemann, E.L. Tomusiak, Eur. Phys. J. A23, 147-190 (2005)

[4] D. Rozpȩdzik, PhD thesis, Krakow (2010); D. Rozpedzik, et al., Phys. Rev. C83, 064004 (2011)

[5] J.D. Bjorken and S.D. Drell, Relativistic Quantum Mechanics, McGraw-Hill Book. Company, New York (1964)

[6] W. Leidemann, E.L. Tomusiak, H. Arenhövel, Phys. Rev. C43, 1022 (1991) 